\title{
Exact Global Solutions of Brane Universes and Big Bounce
}

\author{
Hongya Liu* \\ Department of Physics, Dalian University of Technology, Dalian 116024, P.R. China
}

\begin{abstract}
Exact global solutions of five-dimensional cosmological models compactified on a $S_{1} / Z_{2}$ orbifold with two 3-branes are presented, and evolution of a simple model is studied. It is found that on all $4 \mathrm{D}$ spacetime hypersurfaces, except a singular one, the expanding universe was started not from a big bang but from a big bounce, and before the bounce the universe was in a deflationary contracting phase. It is also found that whether the energy density on the second brane is positive, zero, or negative depends on the size of the fifth dimension.
\end{abstract}

PACS numbers: 04.50.+h; 11.10.Kk; 98.80.Cq.

*Electronic address: hyliu@dlut.edu.cn 
It was proposed [1-3] that our universe is a 3-brane embedded in a higher dimensional space. While gravity can freely propagate in all dimensions, the standard matter particles and forces are confined to the 3-brane. Binetruy, Deffayet and Langlois (BDL) have considered a 5D cosmological model and derived the Friedmann equations on the branes [4]. This model has received extensive studies and most of these studies were focused on the branes only [5]. Be aware that it is technically difficult to move from the branes and construct exact solutions of the field equations in the bulk, whereas numerous exact solutions are known in 5D Kaluza-Klein theories. This suggests that we can take a solution of the Kaluza-Klein theory and use the $Z_{2}$ reflection symmetry and Israel's jump conditions to obtain a global brane model. In what follows, we will do this for a rich class of 5D cosmological solutions found originally by Liu and Mashhoon [6] and restudied recently by Liu and Wesson $[7,8]$. We will then construct a class of global brane solutions which is of the simplest BDL'type, i.e., the matter on the two branes is a perfect fluid, the bulk is empty, and no cosmological constants are added in the bulk and on the branes.

The $5 \mathrm{D}$ metric for the solutions is

$$
d S^{2}=B^{2} d t^{2}-A^{2}\left(\frac{d r^{2}}{1-k r^{2}}+r^{2} d \Omega^{2}\right)-d y^{2} .
$$

where $B=B(t, y)$ and $A=A(t, y)$ are two scale factors, $k(= \pm 1$ or 0$)$ is the $3 \mathrm{D}$ curvature index, and $d \Omega^{2} \equiv\left(d \theta^{2}+\sin ^{2} \theta d \phi^{2}\right)$. A class of general solutions of the $5 \mathrm{D}$ vacuum equations

$$
R_{A B}=0, \quad A, B=0123 ; 5
$$

was given [7] by

$$
A^{2}=\left(\mu^{2}+k\right) y^{2}+2 \nu y+\frac{\nu^{2}+K}{\mu^{2}+k}, \quad B=\frac{1}{\mu} \frac{\partial A}{\partial t} \equiv \frac{\dot{A}}{\mu},
$$

where an overdot denotes partial derivative with respect to $t, \mu=\mu(t)$ and $\nu=\nu(t)$ are two arbitrary functions, and $K$ is a $5 \mathrm{D}$ curvature constant related to the square of the Riemann-Christoffel tensor (Kretschmann scalar) via

$$
R_{A B C D} R^{A B C D}=\frac{72 K^{2}}{A^{8}} .
$$

To confirm solutions (3), one can go to see the detailed derivation of the solutions in Ref. [6] (in which different notations were used); or one can substitute (3) into $R_{A B}$ directly (which is complicated); or one can use computer programs such as MAPLE or GRTensor. 
Because solutions (3) satisfy the 5D vacuum equations (2), we can use them as the bulk solutions of the BDL-type brane models. Note that one can change $y$ to $-y$ without violating the validity of (3) as exact solutions of (2). Therefore, to obtain brane models we use the $Z_{2}$ reflection symmetry on (3), i.e., we let

$$
A^{2}=\left(\mu^{2}+k\right) y^{2}-2 \nu|y|+\frac{\nu^{2}+K}{\mu^{2}+k}, \quad B=\frac{1}{\mu} \frac{\partial A}{\partial t} \equiv \frac{\dot{A}}{\mu} .
$$

Then we take the corresponding 5D Einstein equations being

$$
\begin{aligned}
G_{A B}= & k_{(5)}^{2} T_{A B} \\
T_{B}^{A}= & \delta(y) \operatorname{diag}\left(\rho_{1},-p_{1},-p_{1},-p_{1}, 0\right) \\
& +\delta\left(y-y_{2}\right) \operatorname{diag}\left(\rho_{2},-p_{2},-p_{2},-p_{2}, 0\right)
\end{aligned}
$$

Here the first brane is at $y=y_{1}=0$ and the second is at $y=y_{2}>0$. In the bulk we have $T_{A B}=0$ and then $G_{A B}=0$, so equations (6) are satisfied by (5). On the branes we have to solve the equations ([6]) as follows.

We use the 5D metric (II) to calculate the 5D Einstein tensor, which, substituted in (6), gives

$$
\begin{gathered}
G_{00}=3\left(\frac{\dot{A}^{2}}{A^{2}}+k \frac{B^{2}}{A^{2}}\right)-3 B^{2}\left(\frac{A^{\prime \prime}}{A}+\frac{A^{2}}{A^{2}}\right) \\
=k_{(5)}^{2} B^{2}\left[\rho_{1} \delta(y)+\rho_{2} \delta\left(y-y_{2}\right)\right] \\
G_{05}=-3\left(\frac{\dot{A}^{\prime}}{A}-\frac{\dot{A}}{A} \frac{B^{\prime}}{B}\right)=0 \\
\left(1-\frac{3}{B^{2}}\left[\frac{\ddot{A}}{A}+\frac{\dot{A}}{A}\left(\frac{\dot{A}}{A}-\frac{\dot{B}}{B}\right)+k \frac{B^{2}}{A^{2}}\right]+\frac{3 A^{\prime}}{A}\left(\frac{A^{\prime}}{A}+\frac{B^{\prime}}{B}\right)=0\right. \\
=-\frac{A^{2}}{B^{2}}\left[\frac{2 \ddot{A}}{A}+\frac{\dot{A}}{A}\left(\frac{\dot{A}}{A}-\frac{2 \dot{B}}{B}\right)+k \frac{B^{2}}{A^{2}}\right] \\
+A^{2}\left[\frac{B^{\prime \prime}}{B}+\frac{2 A^{\prime \prime}}{A}+\frac{A^{\prime}}{A}\left(\frac{A^{\prime}}{A}+\frac{2 B^{\prime}}{B}\right)\right] \\
=k_{(5)}^{2} A^{2}\left[p_{1} \delta(y)+p_{2} \delta\left(y-y_{2}\right)\right] .
\end{gathered}
$$


According to Israel's jump conditions, the two scale factors $A$ and $B$ are required to be continuous across the two branes. Their first derivatives with respect to $y$ can be discontinuous across the branes, and then their second derivatives give a Dirac delta function. So we have to calculate $A^{\prime}$ and $B^{\prime}$ across the two branes. By differentiating $A^{2}$ in (5) with respect to $y$, we obtain

$$
A A^{\prime}=\left(\mu^{2}+k\right) y-\nu \frac{\partial|y|}{\partial y}
$$

Therefore we get

$$
\begin{aligned}
& A^{\prime}\left(0^{+}\right)=-\frac{\nu}{A_{1}}, \quad A^{\prime}\left(0^{-}\right)=\frac{\nu}{A_{1}}, \\
& A^{\prime}\left(y_{2}^{+}\right)=-\frac{\mu^{2}+k}{A_{2}} y_{2}+\frac{\nu}{A_{2}}, \quad A^{\prime}\left(y_{2}^{-}\right)=\frac{\mu^{2}+k}{A_{2}} y_{2}-\frac{\nu}{A_{2}},
\end{aligned}
$$

where $A_{1} \equiv A\left(t, y=y_{1}=0\right)$ and $A_{2} \equiv A\left(t, y=y_{2}\right)$. Then, using (12) and the second equation in (5), i.e., $B=\dot{A} / \mu$, we get

$$
\begin{aligned}
& B^{\prime}\left(0^{+}\right)=-\frac{1}{\mu} \frac{\partial}{\partial t}\left(\frac{\nu}{A_{1}}\right), \quad B^{\prime}\left(0^{-}\right)=\frac{1}{\mu} \frac{\partial}{\partial t}\left(\frac{\nu}{A_{1}}\right) \\
& B^{\prime}\left(y_{2}^{+}\right)=-\frac{1}{\mu} \frac{\partial}{\partial t}\left(\frac{\mu^{2}+k}{A_{2}} y_{2}-\frac{\nu}{A_{2}}\right), \quad B^{\prime}\left(y_{2}^{-}\right)=\frac{1}{\mu} \frac{\partial}{\partial t}\left(\frac{\mu^{2}+k}{A_{2}} y_{2}-\frac{\nu}{A_{2}}\right) .
\end{aligned}
$$

So the jumps of $A^{\prime}$ and $B^{\prime}$ across the two branes are

$$
\begin{aligned}
& {\left[A^{\prime}\right]_{1}=-\frac{2 \nu}{A_{1}}, \quad\left[A^{\prime}\right]_{2}=-2\left(\frac{\mu^{2}+k}{A_{2}} y_{2}-\frac{\nu}{A_{2}}\right),} \\
& {\left[B^{\prime}\right]_{1}=-\frac{2}{\mu} \frac{\partial}{\partial t}\left(\frac{\nu}{A_{1}}\right), \quad\left[B^{\prime}\right]_{2}=-\frac{2}{\mu} \frac{\partial}{\partial t}\left(\frac{\mu^{2}+k}{A_{2}} y_{2}-\frac{\nu}{A_{2}}\right),}
\end{aligned}
$$

where $\left[A^{\prime}\right]_{1} \equiv A^{\prime}\left(0^{+}\right)-A^{\prime}\left(0^{-}\right)$and so on. Substituting (14) in the field equations ( 7 ) and (10), we obtain

$$
\begin{aligned}
& k_{(5)}^{2} \rho_{1}=-\frac{3}{A_{1}}\left[A^{\prime}\right]_{1}=\frac{6 \nu}{A_{1}^{2}}, \\
& k_{(5)}^{2} p_{1}=\frac{1}{B_{1}}\left[B^{\prime}\right]_{1}+\frac{2}{A_{1}}\left[A^{\prime}\right]_{1}=-\frac{2}{\dot{A}_{1}} \frac{\partial}{\partial t}\left(\frac{\nu}{A_{1}}\right)-\frac{4 \nu}{A_{1}^{2}},
\end{aligned}
$$

and

$$
\begin{aligned}
k_{(5)}^{2} \rho_{2} & =-\frac{3}{A_{2}}\left[A^{\prime}\right]_{2}=\frac{6}{A_{2}}\left(\frac{\mu^{2}+k}{A_{2}} y_{2}-\frac{\nu}{A_{2}}\right) \\
k_{(5)}^{2} p_{2} & =\frac{1}{B_{2}}\left[B^{\prime}\right]_{2}+\frac{2}{A_{2}}\left[A^{\prime}\right]_{2} \\
& =-\frac{2}{\dot{A}_{2}} \frac{\partial}{\partial t}\left(\frac{\mu^{2}+k}{A_{2}} y_{2}-\frac{\nu}{A_{2}}\right)-\frac{4}{A_{2}}\left(\frac{\mu^{2}+k}{A_{2}} y_{2}-\frac{\nu}{A_{2}}\right) .
\end{aligned}
$$


Thus we have derived the energy densities and pressures on the two branes. Meanwhile, the conservation law $T_{A ; B}^{B}=0$ gives

$$
\dot{\rho}_{i}+3\left(\rho_{i}+p_{i}\right) \frac{\dot{A}_{i}}{A_{i}}=0, \quad i=1,2 .
$$

This relation can also be verified directly by substituting (15), (16) and (15) into (17) as expected.

From the 5D metric (1D) we see that on a given $y=$ constant 4D hypersurface the proper time can be defined as $d \tau=B(t, y) d t$. So the Hubble and deceleration parameters can be defined as

$$
H(t, y) \equiv \frac{1}{B} \frac{\dot{A}}{A}=\frac{\mu}{A}, \quad q(t, y) \equiv-\frac{A}{B} \frac{\partial}{\partial t}\left(\frac{\dot{A}}{B}\right) /\left(\frac{\dot{A}}{B}\right)^{2}=-\frac{A \dot{\mu}}{\mu \dot{A}}
$$

where we have used the relation $B=\dot{A} / \mu$. Meanwhile, the first equation in (5D) for the branes can be written as

$$
\left(\mu^{2}+k\right) A_{i}^{2}=\left[\left(\mu^{2}+k\right) y_{i}-\nu\right]^{2}+K, \quad i=1,2 .
$$

Then, using (15), (16) and (18) in (19), we obtain

$$
\begin{gathered}
H_{i}^{2}+\frac{k}{A_{i}^{2}}=\frac{k_{(5)}^{4}}{36} \rho_{i}^{2}+\frac{K}{A_{i}^{4}}, \quad i=1,2, \\
H_{i}^{2}\left(1-2 q_{i}\right)+\frac{k}{A_{i}^{2}}=-\frac{k_{(5)}^{4}}{12} \rho_{i}\left(\rho_{i}+2 p_{i}\right)-\frac{K}{A_{i}^{4}}, \quad i=1,2 .
\end{gathered}
$$

These two equations are the induced Friedmann equations on the branes.

Thus we obtain a complete set of global exact solutions given in (15) and (15)-(21) which contains two arbitrary functions $\mu(t)$ and $\nu(t)$. From the relation $B=\dot{A} / \mu$ and metric (11) we see that the form of $B d t$ is invariant under an arbitrary coordinate transformation $t \rightarrow \widetilde{t}(t)$.. This freedom can be used to fix one of the two functions $\mu(t)$ and $\nu(t)$. Another freedom corresponds, as is in the standard general relativity, to the unspecified equation of state of matter. For a given equation of state $p=p(\rho)$, the two equations in (15) give a constraint that can be used to determine the function, say, $\nu(t)$. So, generally speaking, if the matter content on the first brane is known, then $\mu(t)$ and $\nu(t)$ can be fixed. Then the whole solutions can be fixed too. Then, by (16), if the size $y_{2}$ of the fifth dimension 
is also known, we will know the matter content on the second brane, and this is of great importance.

Using above procedure, we can, in principle, construct particular models such as the matter and radiation dominated models as in the standard FRW cosmology. However, preliminary studies show that they are not mathematically simple. So we leave this study in the future. Here, in this letter, we will use another way to obtain an explicit universe model, that is, we will choose $\mu(t)$ and $\nu(t)$ firstly and then to study the evolution and matter properties. Now we let

$$
k=0, \quad K=\frac{1}{L^{2}}, \quad \nu(t)=\frac{t_{b}}{L t}, \quad \mu(t)=(2 L t)^{-1 / 2},
$$

where $L$ is a constant with a dimension of length and $t_{b}$ (we assume $t_{b}>0$ ) is a critical constant with a dimension of time. In this way the solutions (5) become

$$
\begin{aligned}
& A^{2}=\frac{2 t}{L}\left[1+\left(\frac{|y|-2 t_{b}}{2 t}\right)^{2}\right] \\
& B^{2}=\left[1-\left(\frac{|y|-2 t_{b}}{2 t}\right)^{2}\right]^{2} /\left[1+\left(\frac{|y|-2 t_{b}}{2 t}\right)^{2}\right] .
\end{aligned}
$$

So on the first brane we have

$$
\begin{aligned}
A_{1}^{2} & =\frac{2 t}{L}\left[1+\left(\frac{t_{b}}{t}\right)^{2}\right], \quad B_{1}^{2}=\left[1-\left(\frac{t_{b}}{t}\right)^{2}\right]^{2}\left[1+\left(\frac{t_{b}}{t}\right)^{2}\right]^{-1}, \\
k_{(5)}^{2} \rho_{1} & =\frac{3 t_{b}}{t^{2}+t_{b}^{2}}, \quad k_{(5)}^{2} p_{1}=\frac{2 t_{b}}{t^{2}-t_{b}^{2}}-\frac{t_{b}}{t^{2}+t_{b}^{2}} .
\end{aligned}
$$

On the second brane we have

$$
\begin{aligned}
A_{2}^{2} & =\frac{2 t}{L}\left[1+\left(\frac{y_{2}-2 t_{b}}{2 t}\right)^{2}\right], \quad B_{2}^{2}=\left[1-\left(\frac{y_{2}-2 t_{b}}{2 t}\right)^{2}\right]^{2}\left[1+\left(\frac{y_{2}-2 t_{b}}{2 t}\right)^{2}\right]^{-1} \\
k_{(5)}^{2} \rho_{2} & =\frac{6\left(y_{2}-2 t_{b}\right)}{4 t^{2}+\left(y_{2}-2 t_{b}\right)^{2}}, \quad k_{(5)}^{2} p_{2}=\frac{4\left(y_{2}-2 t_{b}\right)}{4 t^{2}-\left(y_{2}-2 t_{b}\right)^{2}}-\frac{2\left(y_{2}-2 t_{b}\right)}{4 t^{2}+\left(y_{2}-2 t_{b}\right)^{2}} .
\end{aligned}
$$

Equations (23)-(25) constitute a quite simple two-brane model.

From (23) we see that, in a $y=$ constant hypersurface, the scale factors $B \approx 1$ and $A \approx \sqrt{2 t / L}$ for $2 t \gg|| y\left|-2 t_{b}\right|$. So it approaches the standard radiation-dominated model at late times of the universe. The global evolution of the scale factor $A(t, y)$ with $t_{b}=1$ and $L=1$ is plotted in Fig.1. From this figure we see that there is a singular surface $|y|=2 t_{b}$ in which $A \rightarrow 0$ as $t \rightarrow 0$, showing a big bang singularity as is in the standard 


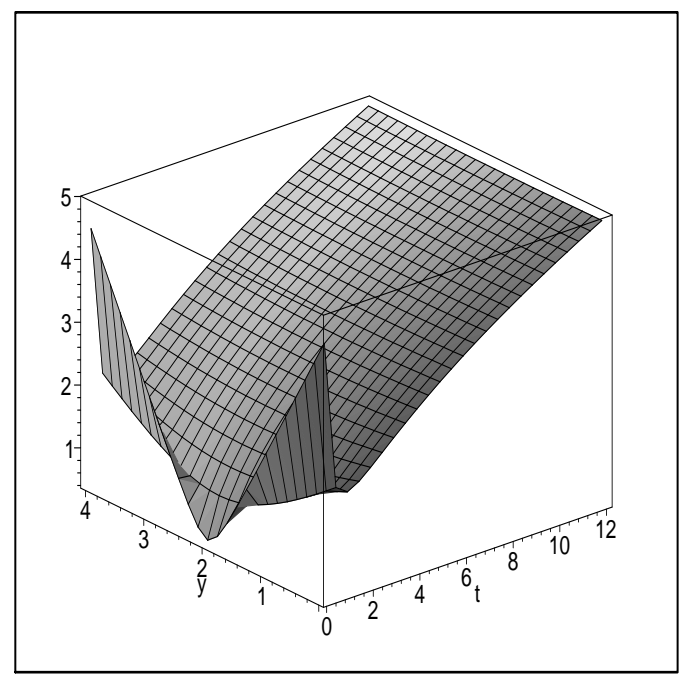

FIG. 1: Global evolution of the scale factor $A(t, y)=\sqrt{2 t+(|y|-2)^{2} /(2 t)}$. The first brane (our universe) is at $y=0$; while the second brane (the hidden universe) could be placed at $y=2$, $0<y<2$, or $y>2$, giving three types of brane cosmological models, respectively.

FRW models. However, in all other hypersurfaces, the scale factor $A$ reaches a non-zero minimum $\sqrt{2 t / L}$ at $2 t=|| y\left|-2 t_{b}\right|$ (where $B=0$ ); and at both sides of this minimum, $A$ tends to infinity. So this minimum can naturally be explained as a big bounce [7]. Generally, the solution has two kinds of singularities, corresponding to $A=0$ and $B=0$ respectively as discussed in Ref. [7]. $A=0$ is, by (44), an intrinsic singularity of the 5D manifold, whereas $B=0$ is just a coordinate singularity similar to the Schwarzschild event horizon. So here the big bounce singularity belongs to the second kind.

The first brane is at $y=0$. By (24) we see that at the bounce point $t=t_{b}$ we have $A_{1}$ tends to its minimum, $B_{1}$ tends to zero, $\rho_{1}$ is finite, and $p_{1}$ tends to infinity. Physically, we can say that it is this infinitely large pressure $p_{1}$ that caused the big bounce.

From (25) we see that the evolution and the matter properties on the second brane depend on the size $y_{2}$ of the fifth dimension (see Fig. 1 also). Thus we have three types of brane models as shown below.

Type I. The second brane is at $y_{2}=2 t_{b}$. Then (25) gives $A_{2}=\sqrt{2 t / L}, B_{2}=1$, and $\rho_{2}=$ $p_{2}=0$. This implies that the second brane is empty of matter. So Type I is actually a one-brane model for which there was a big bounce on our side of the model and a big bang on the "hidden" side. 
Type II. The second brane is at $0<y_{2}<2 t_{b}$. Then (25) shows that there were bounces on both branes. On the second brane we have $\rho_{2}<0$. So Type II is a two-brane model for which the energy density on the second brane is negative.

Type III. The second brane is at $y_{2}>2 t_{b}$. This is also a two-brane model for which the energy density on the second brane is positive. Specifically, if $y_{2}=4 t_{b}$, then $A_{2}=A_{1}$, $B_{2}=B_{1}, \rho_{2}=\rho_{1}$, and $p_{2}=p_{1}$, giving a completely symmetric two-brane model.

Now let us consider the solution (24) for which when the coordinate time $t$ varies from 0 to $t_{b}$ and then to $+\infty$, the spatial scale factor $A_{1}(t)$ contracts from $+\infty$ to a minimum $A_{\min }=2 \sqrt{t_{b} / L}>0$ and then expands to $+\infty$ again. We also find that as $t \rightarrow 0, A_{1}^{2} \rightarrow$ $2 t_{b}^{2} /(L t), B_{1}^{2} \rightarrow\left(t_{b} / t\right)^{2}$ and the $4 \mathrm{D}$ metric on the first brane tends to

$$
d s_{1}^{2} \rightarrow\left(\frac{t_{b}}{t}\right)^{2} d t^{2}-\frac{2 t_{b}^{2}}{L t}\left(d r^{2}+r^{2} d \Omega^{2}\right) \quad(\text { as } t \rightarrow 0) .
$$

By a coordinate transformation

$$
t=2 L^{-1} t_{b}^{2} e^{\tau / t_{b}}
$$

we get

$$
d s_{1}^{2} \rightarrow d \tau^{2}-e^{-\frac{\tau}{t_{b}}}\left(d r^{2}+r^{2} d \Omega^{2}\right) \quad(\text { as } \tau \rightarrow-\infty)
$$

which represents a deflationary de Sitter cosmological model. Note that by (27) $t=0$ corresponds to $\tau=-\infty$. Therefore we conclude that according to the proper time $\tau$, the universe on the first brane has been existed forever and contracts from a de Sitter space to a non-zero minimum, at which the pressure $p_{1}$ reaches to infinity and causes a big bounce. After then, the universe expands.

In conclusion, we have derived a class of exact global solutions of five-dimensional cosmological models with two 3-branes, for which matter on the branes is of the form of a perfect fluid, the bulk is empty, and no cosmological constants were introduced. This solutions contain two arbitrary constants $k$ and $K$, corresponding to the 3D and 5D curvatures respectively, and two arbitrary functions $\mu(t)$ and $\nu(t)$, corresponding to the two freedoms: the arbitrary coordinate transformation $t \rightarrow \widetilde{t}(t)$ and the unspecified equation of state of matter. By choosing $\mu(t)$ and $\nu(t)$ properly a special model is discussed in detail. It is found that in the late times of the universe this model evolves at the same rate as is in the standard radiation-dominated FRW model. And there is a singular 4D hypersurface $y=0$ 
on which there was a big bang. On all other hypersurfaces there were no big bang but big bounces. Before the bounces, the universe was in a contracting phase. It is also found that whether the energy density on the second brane is positive, zero, or negative depend on the size of the fifth dimension. Thus three types of models are obtained for which type I is actually a one-brane model while types II and III are two-brane models with negative and positive energy densities, respectively.

\section{ACKNOWLEDGMENTS}

We thank Paul Wesson and Guowen Peng for comments. This work was supported by NSF of P. R. China under Grants 19975007 and 10273004.

[1] N. Arkani-Hamed, S. Dimopoulos and G. Dvali, Phys. Lett. B 429, 263 (1998), hep-ph/9803315; Phys. Rev. D 59, 086004 (1999), hep-ph/9807344 I. Antoniadis, N. Arkani-Hamed, S. Dimopoulos and G. Dvali, Phys. Lett. B 436, 257 (1998), hep-ph/9804398.

[2] P. Horava and E. Witten, Nucl. Phys. B 460, 506 (1996), hep-th/9510209 E. Witten, Nucl. Phys. B 471, 135 (1996), hep-th/9602070 P. Horava and E. Witten, Nucl. Phys. B. 475, 94 (1996), hep-th/9603142.

[3] L. Randall and R. Sundrum, Phys. Rev. Lett. 83, 3370 (1999), hep-ph/9905221; Phys. Rev. Lett. 83, 4690 (1999), hep-th/9906064.

[4] P. Binetruy, C. Deffayet and D. Langlois, Nucl. Phys. B 565, 269 (2000), hep-th/9905012

[5] See, for example, J.M. Cline, C. Grojean, and G. Servant, Phys. Rev. Lett. 83, 4245 (1999), hep-ph/9906523 C. Csaki, M. Graesser, C. Kolda, and J. Terning, Phys. Lett. B 462, 34 (1999), hep-ph/9906513 P. Kanti, I. Kogan, K.A. Olive and M. Pospelov, Phys. Lett. B 468, 31 (1999), hep-ph/9909481, Phys. Rev. D 61, 106004 (2000), hep-ph/9912266, S. Mukohyama, T. Shiromizu and K. Maeda, Phys. Rev. D 62, 024028 (2000), hep-th/9912287; S. Mukohyama, Phys. Lett. B 473, 241 (2000), hep-th/9911165 J. Khoury and R.-J. Zhang, Phys. Rev. Lett. 89, 061302 (2002), hep-th/0203274.

[6] Hongya Liu and B. Mashhoon, Ann. Phys. (Leipzig) 4, 565 (1995).

[7] Hongya Liu and P.S. Wesson, Astrophys. J. 562, 1 (2001), gr-qc/0107093. 
[8] J. Ponce de Leon, Mod. Phys. Lett. A. 16, 2291 (2001), gr-qc/0111011. 